\title{
Comprehensive Serum Lipidomics for Detecting Incipient Dementia in Parkinson's Disease
}

\section{Supplementary Material}

\section{Appendix A: Materials and Methods}

Adriana Zardini Buzatto ${ }^{1}$, Jaspaul Tatlay ${ }^{1}$, Barinder Bajwa ${ }^{1}$, Dorothea Mung ${ }^{1}$, Richard Camicioli ${ }^{2,3}$, Roger A. Dixon ${ }^{2,4}$ and Liang $\mathrm{Li}^{1 *}$

${ }^{1}$ Department of Chemistry, University of Alberta, Edmonton, AB, T6G 2R3, Canada

${ }^{2}$ Neuroscience and Mental Health Institute, University of Alberta, Edmonton, AB, T6G 2E1, Canada

${ }^{3}$ Department of Medicine (Neurology), University of Alberta, Edmonton, AB, T6G 2G3, Canada

${ }^{4}$ Department of Psychology, University of Alberta, Edmonton, AB, T6G 2R3, Canada

*Corresponding author: Dr. Liang Li

University of Alberta, Department of Chemistry, Room W3-39C

Edmonton, AB, Canada - T6G 2G2

Email: liang.li@ualberta.ca, Telephone: +1 (780) 492-3250 


\section{Contents}

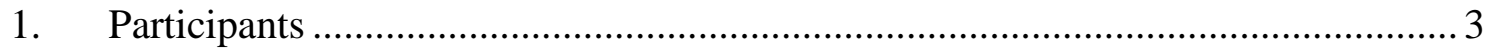

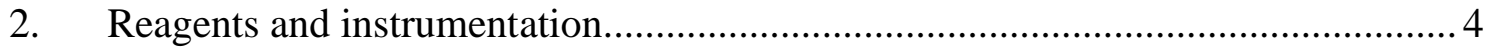

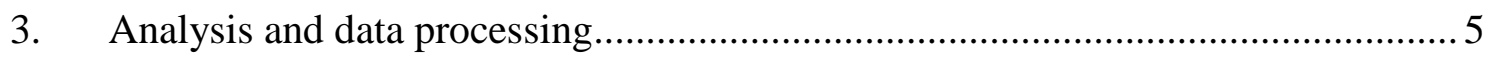

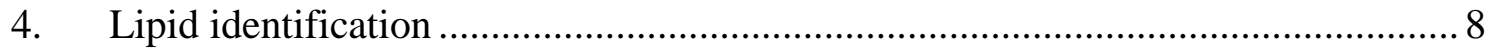

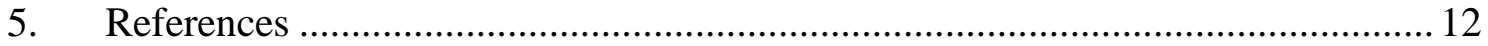




\section{Participants}

We employed serum samples from 43 PD patients and 43 healthy controls that were enrolled in our previously published works. ${ }^{1-4}$ Aliquots of each serum sample were archived at $80^{\circ} \mathrm{C}$ for further analysis, including the present study. All samples were kept under identical conditions and for the same period to prevent bias. PD patients were recruited from the University of Alberta Movement Disorder Clinics, community neurologists, and the Parkinson's Society of Alberta (Edmonton, AB, Canada). Patients had no features of atypical Parkinsonism and no unstable health conditions compromising survival. The 43 healthy controls aged between 65 and 84 years old were recruited via seniors' centers, advertisements, clinics, and patient contacts.

No patients or controls displayed cognitive deficits at the time of sample collection (baseline, 0 months). Volunteers were reassessed after 18 and 36 months for cognitive and functional decline by the study neurologist (Dr. Richard Camicioli, University of Alberta, Canada) and a research assistant through interviews with patients and caregivers, as well as cognitive and functional tests (Supp. Table 1). Each visit included evaluation of the standardized Mini-Mental Status Examination (MMSE), Frontal Assessment Battery (FAB), Dementia Rating Scale (DRSII), Clinical Dementia Rating (CDR), Geriatric Depression Scale (GDS), Blessed-OrientationConcentration Test, Unified Parkinson's Disease Rating Scale (UPDRS), Hoehn and Yahr Rating

Scale (HY), and Cumulative Illness Rating Scale (CIRS). ${ }^{1-3}$ Demographic information (age, sex, education), vital signs, Levodopa-equivalent dosages and other medications were also recorded (Supp. Table 1). Patients that did not meet criteria for PD or that developed other conditions in the follow-up visits were excluded before the analysis phase, as well as any participants with baseline dementia (CDR <1.0; MMSE <23), stroke, cerebellar signs, early autonomic dysfunction, supranuclear gaze palsy, conditions that might affect cognition (recent myocardial infarction, 
angina, congestive heart failure), severe depression, psychosis, cognitive sequelae of head trauma, alcoholism, drug abuse, or atypical parkinsonism. No volunteers were taking cognitive enhancing drugs during the study. ${ }^{1-4}$

Parkinson's disease dementia was defined 36 months after sample collection by a significant cognitive and functional decline in at least two criteria, i.e., a 3-point change on the MMSE, 6-point change on the DRS-II, or impairment in two or more domains on the CDR when compared to baseline during the 3 years, along with functional impairment due to cognitive decline. All PD patients were cognitively stable at the moment of sample collection (i.e., no clinical symptoms of dementia) but part of them were diagnosed with dementia at a regularly scheduled assessment 36 months after baseline. Hence, the PD patients that were later diagnosed with dementia are referred to as Parkinson's disease with incipient dementia (PDID). Patients that remained cognitively stable during the 36-month interval of this study are referred to as Parkinson's disease with no dementia (PDND). Further information for the definition of dementia can be found in our related work. ${ }^{1-4}$

\section{Reagents and instrumentation}

Chemicals and reagents were purchased from Sigma-Aldrich (St. Louis, MO, USA; ammonium formate), Avanti Polar Lipids (Alabaster, AL, USA; Splash Lipidomix Mass Spec Standard) and Fisher Scientific (Waltham, MA, USA; analytical grade dichloromethane and LCMS grade methanol, 2-propanol, acetonitrile and water). Samples were analyzed by reversed-phase liquid chromatography (RP-LC) using a Dionex UltiMate 3000 UHPLC system (Thermo Fisher Scientific, Waltham, MA, USA) with a Waters Acquity BEH C18 column $(5 \mathrm{~cm} \times 2.1 \mathrm{~mm}$ with 
$1.7 \mu \mathrm{m}$ particles; Waters Corporation, Milford, MA, USA) and an ultra-high resolution Maxis II Quadrupole Time-of-Flight (QToF) mass spectrometer (Bruker Daltonics, Billerica, MA, USA) equipped with an electrospray ionization (ESI) source.

\section{Analysis and data processing}

Blood serum samples were prepared according to a modified Folch liquid-liquid extraction protocol with a combination of dichloromethane, methanol and water. The classical Folch method describes the extraction of lipids from at least $1 \mathrm{~g}$ of homogenized tissue samples with 20 parts of 2:1 chloroform/methanol (v/v), followed by clean-up step with 0.2 parts of water, i.e., a final ratio of 8:4:3 chloroform/ methanol/ water. ${ }^{5,6}$ This liquid-liquid extraction method is widely used by the lipidomics community due to comprehensive coverage and suitable recoveries for most lipid subclasses. ${ }^{7-12}$ For this work, the solvent volumes were adapted and optimized to reduced volumes of serum samples ( $6.0 \mu \mathrm{L}$ for positive ionization and $30.0 \mu \mathrm{L}$ for negative ionization). Chloroform was substituted by dichloromethane due to a lower carcinogenic risk, fewer restrictions to acquisition and lower cost. ${ }^{13,14}$ The low polarity of dichloromethane allows the selective extraction of lipids into the organic phase, while the combination with methanol adds a better control of partition coefficients for compounds with intermediate polarity, as well as disruption of lipid aggregates and protein precipitation. Finally, the water wash step guarantees clean-up of the organic phase, removing hydrophilic molecules that may be trapped by lipid aggregates. ${ }^{12,15,16}$

Samples were thawed in a $4^{\circ} \mathrm{C}$ refrigerator for $30 \mathrm{~min}$ before extraction. Different sample volumes and final dilutions were employed for detection under positive and negative electrospray ionization due to the inherent characteristics of each polarity. For positive ionization, aliquots of 
$6.0 \mu \mathrm{L}$ of human serum were vortexed with $2.0 \mu \mathrm{L}$ of the internal standard mixture (Splash Lipidomix Mass Spec Standard, Avanti Polar Lipids), 54.0 $\mu \mathrm{L}$ of methanol, 111.0 $\mu \mathrm{L}$ of dichloromethane and $28.0 \mu \mathrm{L}$ of water. After a 10 min equilibration at room temperature, samples were centrifuged for $10 \mathrm{~min}$ at $10,000 \mathrm{rpm}$ and $4^{\circ} \mathrm{C}$. The bottom organic layer was evaporated to dryness using a SpeedVac for $30 \mathrm{~min}$. The residue was immediately resuspended in $12.0 \mu \mathrm{L}$ of 1:1 mobile phase A (MPA) / mobile phase B (MPB) and diluted with $108.0 \mu \mathrm{L}$ of MPA (20-fold dilution). For negative ionization, $30.0 \mu \mathrm{L}$ aliquots of serum and $10.0 \mu \mathrm{L}$ of the internal standard mixture were extracted with $46.0 \mu \mathrm{L}$ of methanol, $111.0 \mu \mathrm{L}$ of dichloromethane and $4.0 \mu \mathrm{L}$ of water, followed by resuspension in $3.0 \mu \mathrm{L}$ of 1:1 MPA/MPB and dilution with $27.0 \mu \mathrm{L}$ of MPA (no dilution). All samples were injected within $48 \mathrm{~h}$ after extraction. The extracts were kept at $4^{\circ} \mathrm{C}$ in $250 \mu \mathrm{L}$ polypropylene inserts (Canadian Life Science, Peterborough, ON, Canada) placed inside $2 \mathrm{~mL}$ amber vials (Agilent Technologies, Santa Clara, CA, USA) with PTFE/silicone septa caps (Waters Corporation, Milford, MA, USA) until injection. We recognize that the use of plastic ware with organic solvents is not ideal, although much more practical and affordable than glassware. ${ }^{12}$ For this study, all samples were prepared using the same type and lot numbers of plastic materials (inserts, septa, vial caps and pipet tips), thus minimizing the effects of plasticizers and other contaminants upon the statistical analysis. Blank extractions of water instead of the sample were performed with each sample batch to ensure quality control. All samples were analyzed with experimental duplicates, and samples were randomized for preparation and analysis.

Samples were analyzed by RP-LC-MS with a C18 chromatographic column using MPA $10 \mathrm{mM}$ ammonium formate in 50:40:10 methanol/ acetonitrile/ water; $\mathrm{MPB}-10 \mathrm{mM}$ ammonium formate in 95:5 2-propanol/ water; $250 \mu \mathrm{L} / \mathrm{min}$; column temperature of $40^{\circ} \mathrm{C}$; injection volume of $5.0 \mu \mathrm{L}$ for positive ionization and $9.0 \mu \mathrm{L}$ for negative ionization; and $22 \mathrm{~min}$ gradient $(0 \mathrm{~min}-5 \%$ 
MPB; $1.8 \min -5 \% \mathrm{MPB} ; 8.5 \min -30 \% \mathrm{MPB} ; 18 \min -95 \% \mathrm{MPB} ; 22 \min -95 \% \mathrm{MPB})$ followed by 10 min of equilibration ( 0 min $-95 \%$ MPB; $3 \min -95 \%$ MPB; 4 min - 5\% MPB; 10 min $-5 \%$ MPB). The high hydrophobicity of most lipid species requires strong organic solvents for elution; therefore, the combination of acetonitrile, methanol and water provides high selectivity through hydrogen bonding and $\pi$ interactions, whereas the low polarity of 2-propanol guarantees the solubilization and elution of long-chain, hydrophobic lipids. Moreover, most lipid species are not easily ionized under soft ionization techniques and can only be detected as adducts. For this work, ammonium formate was employed as an additive on both mobile phases to promote adduct formation, increasing the detection of different lipid classes.

A QToF mass spectrometer instrument equipped with an ESI source was used for detection under positive and negative ionization (capillary voltage of $4500 \mathrm{~V}$; endplate offset of $500 \mathrm{~V}$, nebulizer gas pressure of 1.0 bar, dry gas flow rate of $8.0 \mathrm{~L} / \mathrm{min}$; dry temperature of $230^{\circ} \mathrm{C}$; spectra acquisition rate of $1 \mathrm{~Hz}, \mathrm{~m} / \mathrm{z}$ range of 150 to $1500 \mathrm{Da}$ ). A 1.5 min segment for mass re-calibration was inserted at the beginning of each chromatogram during which $0.10 \mathrm{mM}$ sodium formate calibrant solution was infused into the ion source using a peristaltic pump. The characteristic sodium formate clusters were further employed to correct the mass/charge $(\mathrm{m} / \mathrm{z})$ values detected throughout each chromatogram, improving mass accuracy. We also employed a lock mass recalibration using erucamide $(\mathrm{m} / \mathrm{z} 338.34173)$ for positive ionization and stearic acid for negative ionization ( $\mathrm{m} / \mathrm{z} 283.26425)$, common contaminants detected throughout all injections.

Chromatograms were processed using MetaboScape 4.0 (Bruker Daltonics, Billerica, MA, USA) with minimum intensity cut-off of 4000 cts for positive and 2000 cts for negative ionization; minimum peak length of 6 spectra; retention time tolerance of $15 \mathrm{~s} ; \mathrm{m} / \mathrm{z}$ tolerance of $5.0 \mathrm{mDa}$; missing value substitution by recursive extraction for features detected in at least $10 \%$ of 
injections; adduct correlation cut-off of 0.75 , and filtering by detection in at least $80 \%$ of injections within at least one group (control, Parkinson's disease with no dementia diagnosis - PDND, Parkinson's disease with incipient dementia-PDID, or QC). Features detected under positive and negative ionization were merged into a unique list.

\section{Lipid identification}

MS/MS identification (tiers 1 and 2) was performed with the MS-Dial LipidBlast MS/MS library (Bruker Corporation), Bruker Human Metabolome Database (HMDB) Metabolite Library 2.0 (Bruker Corporation) and MassBank of North America (MoNA) LC-MS/MS libraries (LipidBlast, FAHFA, Riken IMS Oxidized Phospholipids, EMBL-MCF, MassBank and all LCMS/MS spectra) in combination with MetaboScape 4.0 (Bruker Daltonics, Billerica, MA, USA). 17-24 Abbreviations to lipid classes and nomenclature followed the LipidMaps database (https://www.lipidmaps.org), $\quad$ MS-DIAL LipidBlast spectral library (http://prime.psc.riken.jp/Metabolomics_Software/MS-DIAL/index5.html), and the Lipidomics Standard Initiative (https://lipidomics-standards-initiative.org) (Appendix D, Supp. Table 3). $17,18,25-27$

Features not identified by MS/MS were inputted in the Lipid Maps database for putative mass match (tier 3, tolerance of $5.0 \mathrm{mDa}$ ). ${ }^{28}$ Lipids can have a high number of isomers and isobars, particularly for mass-based identification. A mass search for lipids can define (1) the lipid class, (2) the sum of components, i.e., total number of carbons in fatty acyl/alkyl chains and double bonds, and (3) the bond type. The individual compositions of specific fatty acyl/alkyl chains require MS/MS match, and cannot be determined by a mass search. ${ }^{27}$ Furthermore, 
isomeric/isobaric overlap for different lipid classes and adducts often occurs. Each detected feature can be $\mathrm{m} / \mathrm{z}$ matched to a high number of isomeric and isobaric lipids that may belong to different classes. Hence, we employed a 6-tier filtering and scoring approach to select the best identification possibility for each detected feature. All isomeric and isobaric possibilities that passed initial filters for retention time and adduct detection were kept for each mass-matched feature, but they were ranked according to the characteristics of the employed method, lipid subclass and biological fluids. Low scores were given to the most likely identification possibilities, while high scores were given for less plausible lipids. The lipid with the lowest score was used to determine the subclass of each identified compound.

First, we applied a retention time filter to exclude identification possibilities that could not elute in the detected retention time, e.g., a triacylglycerol is expected to have high retention by the reverse-phase chromatography conditions employed for this work; hence, mass-match identifications for triacylglycerols with low retention times were excluded. We employed lipid standards and high-confidence MS/MS identifications to determine the expected retention time range for each lipid subclass, sub-divided into four fatty acyl length groups (sum of the number of carbons for all fatty acyl residues). The expected retention time ranges were then used to filter out identification possibilities that could not elute in the detected times. The tier 1 and 2 MS/MS identifications also passed through the retention time filter.

Second, we employed a filter for the identified adducts. Most lipid classes are not easily ionized by ESI as the common $[\mathrm{M}+\mathrm{H}]^{+}$and $[\mathrm{M}-\mathrm{H}]^{-}$ions; instead, they are detected as adducts that depend upon modifiers added to the mobile phases and sample medium. The mobile phases and chromatographic conditions used for this work allow different adducts to be detected depending on the structure of each molecule, including $[\mathrm{M}+\mathrm{H}]^{+},\left[\mathrm{M}+\mathrm{NH}_{4}\right]^{+},[\mathrm{M}+\mathrm{Na}]^{+},\left[\mathrm{M}-\mathrm{H}_{2} \mathrm{O}+\mathrm{H}\right]^{+},[\mathrm{M}-\mathrm{H}]^{-}$ 
, $[\mathrm{M}+\mathrm{HCOO}]^{-}$and $\left[\mathrm{M}-\mathrm{CH}_{3}\right]^{-}$. However, different lipid subclasses can form different ions and adducts, e.g., simple fatty acids are often detected as $[\mathrm{M}-\mathrm{H}]^{-}$but triacylglycerols cannot be ionized by the loss of a proton, being more commonly detected in positive ion mode as the $\left[\mathrm{M}+\mathrm{NH}_{4}\right]^{+}$ adduct. Therefore, we also employed lipid standards, high-confidence MS/MS identifications and databases such as LipidMaps to determine the possible adducts for each lipid subclass. ${ }^{28}$ While identification possibilities for adducts that could not be detected were excluded (e.g., triacylglycerols as $[\mathrm{M}-2 \mathrm{H}]^{2-}$ ), all the possible matches were scored according to their expected ionization. The most likely adduct possibilities for each feature were given a score of one, while the less likely possibilities were given higher scores.

Third, the identifications for the same feature were scored by their mass errors. While all identifications had a maximum mass error of $5.0 \mathrm{mDa}$, relative $\mathrm{m} / \mathrm{z}$ errors smaller than $5.0 \mathrm{ppm}$ were given a score of one, while errors between 5.0 and $10.0 \mathrm{ppm}$ received two points and errors higher than 10.0 ppm received three points.

Fourth, the isomeric and isobaric identifications were scored according to an odd or even number of carbons in fatty acyl groups. Even-numbered fatty acyls are more commonly found in mammals due to the acetyl-CoA biosynthesis route, although odd-chain fatty acyl groups can be detected in smaller amounts. ${ }^{29}$ Therefore, identification possibilities for isomers or isobars with even-chain fatty acyls received a score of one, while odd-chain fatty acyls were scored two points (i.e., less plausible).

Fifth, the presence of functional groups other than the expected for each lipid subclass (oxidation, dehydration, heteroatoms, etc.) was scored two points, while the absence of extra groups received one point due to being more common in biological fluids. Although modifications are typical for lipids, they require the action of specific enzymes or processes, leading to lower 
abundance when compared to unmodified species. We also included a score for plasmenyl lipids, which contain vinyl ether bonds that are easily oxidized and degraded. Hence, plasmenyl options received a score of two, while non-plasmenyl lipids were scored one point.

Sixth, each identification possibility was given a score between one and ten to account for the ionization efficiency and sensitivity of the employed methods for each lipid subclass in positive or negative ionization mode. For example, phosphatidylcholines have hydrogen-bond acceptor sites and are easily ionized in positive ion mode using the method employed for this work; hence, the subclass received the best score of one when mass-matched to positive ions. Contrastingly, PCs lack hydrogen-bond donors and can usually only be detected as the formate adduct for negative ionization with distinctive lower peak intensities, rendering a worse (i.e., higher) score of eight when mass-matched to negative ions.

Finally, the scores for each ranking tier were normalized between 0 and 1 to remove biases and received weights according to their importance for lipid identification. The adduct, modifications, plasmenyl and sensitivity scores received a weight of one, while the mass error and even/odd fatty acyl group were given a weight of two. All scores were then summed and the top choice for each feature was selected by the smallest total score. This top choice was used to determine lipid subclass for normalization and during the discussion section, but other isomeric or isobaric possibilities that passed the exclusion filters for retention time range and adducts were also kept, ordered by normalized score values. 


\section{References}

(1) Han, W.; Sapkota, S.; Camicioli, R.; Dixon, R. A.; Li, L. Profiling Novel Metabolic Biomarkers for Parkinson's Disease Using in-Depth Metabolomic Analysis. Mov. Disord. 2017, 32 (12), 1720-1728. https://doi.org/10.1002/mds.27173.

(2) Camicioli, R.; Sabino, J.; Gee, M.; Bouchard, T.; Fisher, N.; Hanstock, C.; Emery, D.; Martin, W. R. R. W. Ventricular Dilatation and Brain Atrophy in Patients with Parkinson's Disease with Incipient Dementia. Mov. Disord. 2011, 26 (8), 1443-1450. https://doi.org/10.1002/mds.23700.

(3) Acharya, H. J.; Bouchard, T. P.; Emery, D. J.; Camicioli, R. M. Axial Signs and Magnetic Resonance Imaging Correlates in Parkinson's Disease. Can. J. Neurol. Sci. / J. Can. des Sci. Neurol. 2007, 34 (1), 56-61. https://doi.org/10.1017/S0317167100005795.

(4) McDermott, K. L.; Fisher, N.; Bradford, S.; Camicioli, R. Parkinson's Disease Mild Cognitive Impairment Classifications and Neurobehavioral Symptoms. Int. Psychogeriatrics 2018, 30 (2), 253-260. https://doi.org/10.1017/S1041610217002265.

(5) Folch, J.; Ascoli, I.; Lees, M.; Meath, J. A.; LeBaron, N. Preparation of Lipide Extracts from Brain Tissue. J. Biol. Chem. 1951, 191 (2), 833-841.

(6) Folch, J.; Lees, M.; Sloane Stanley, G. H. A Simple Method for the Isolation and Purification of Total Lipides from Animal Tissues. J. Biol. Chem. 1957, 226 (1), 497-509.

(7) Ferraz, T. P. L.; Fiúza, M. C.; dos Santos, M. L. A.; Pontes de Carvalho, L.; Soares, N. M. Comparison of Six Methods for the Extraction of Lipids from Serum in Terms of Effectiveness and Protein Preservation. J. Biochem. Biophys. Methods 2004, 58 (3), 187- 
193. https://doi.org/10.1016/j.jbbm.2003.10.008.

(8) Reis, A.; Rudnitskaya, A.; Blackburn, G. J.; Fauzi, N. M.; Pitt, A. R.; Spickett, C. M. A Comparison of Five Lipid Extraction Solvent Systems for Lipidomic Studies of Human LDL. J. Lipid Res. 2013, 54 (7), 1812-1824. https://doi.org/10.1194/jlr.M034330.

(9) Holčapek, M.; Liebisch, G.; Ekroos, K. Lipidomic Analysis. Anal. Chem. 2018, 90 (7), 4249-4257. https://doi.org/10.1021/acs.analchem.7b05395.

(10) Pellegrino, R. M.; Di Veroli, A.; Valeri, A.; Goracci, L.; Cruciani, G. LC/MS Lipid Profiling from Human Serum: A New Method for Global Lipid Extraction. Anal. Bioanal. Chem. 2014, 406 (30), 7937-7948. https://doi.org/10.1007/s00216-014-8255-0.

(11) Bang, D. Y.; Byeon, S. K.; Moon, M. H. Rapid and Simple Extraction of Lipids from Blood Plasma and Urine for Liquid Chromatography-Tandem Mass Spectrometry. J. Chromatogr. A 2014, 1331, 19-26. https://doi.org/10.1016/j.chroma.2014.01.024.

(12) Zardini Buzatto, A.; Kwon, B. K.; Li, L. Development of a NanoLC-MS Workflow for High-Sensitivity Global Lipidomic Analysis. Anal. Chim. Acta 2020, 1139, 88-99. https://doi.org/10.1016/j.aca.2020.09.001.

(13) Chaves, A. L.; Vergara, C. E.; Mayer, J. E. Dichloromethane as an Economic Alternative to Chloroform in the Extraction of DNA from Plant Tissues. Plant Mol. Biol. Report. 1995, 13 (1), 18-25. https://doi.org/10.1007/BF02668389.

(14) Cequier-Sánchez, E.; Rodríguez, C.; Ravelo, A. G.; Zárate, R.; Avelo, N. G. R.; Guez, C. O. R. O. D. R. I.; Sa, F.; Cequier-Sánchez, E.; Rodríguez, C.; Ravelo, A. G.; Zárate, R.; Avelo, N. G. R.; Guez, C. O. R. O. D. R. I.; Sa, F.; Cequier-Sánchez, E.; Rodríguez, C.; 
Ravelo, A. G.; Zárate, R. Dichloromethane as a Solvent for Lipid Extraction and Assessment of Lipid Classes and Fatty Acids from Samples of Different Natures. J. Agric. Food Chem. 2008, 56 (12), 4297-4303. https://doi.org/10.1021/jf073471e.

(15) Zardini Buzatto, A.; Sarkar, I.; Van Drunen Littel-Van Den Hurk, S.; Li, L. Comprehensive Lipidomic and Metabolomic Analysis for Studying Metabolic Changes in Lung Tissue Induced by a Vaccine against Respiratory Syncytial Virus. ACS Infect. Dis. 2020, 6 (8), 2130-2142. https://doi.org/10.1021/acsinfecdis.0c00210.

(16) Zardini Buzatto, A.; Abdel Jabar, M.; Nizami, I.; Dasouki, M.; Li, L.; Abdel Rahman, A. M. Lipidome Alterations Induced by Cystic Fibrosis, CFTR Mutation, and Lung Function. J. Proteome Res. 2021, 20 (1), 549-564. https://doi.org/10.1021/acs.jproteome.0c00556.

(17) Kind, T.; Liu, K.-H.; Lee, D. Y.; DeFelice, B.; Meissen, J. K.; Fiehn, O. LipidBlast in Silico Tandem Mass Spectrometry Database for Lipid Identification. Nat. Methods 2013, 10 (8), 755-758. https://doi.org/10.1038/nmeth.2551.

(18) Tsugawa, H.; Cajka, T.; Kind, T.; Ma, Y.; Higgins, B.; Ikeda, K.; Kanazawa, M.; VanderGheynst, J.; Fiehn, O.; Arita, M. MS-DIAL: Data-Independent MS/MS Deconvolution for Comprehensive Metabolome Analysis. Nat. Methods 2015, 12 (6), 523526. https://doi.org/10.1038/nmeth.3393.

(19) Wishart, D. S.; Feunang, Y. D.; Marcu, A.; Guo, A. C.; Liang, K.; Vázquez-Fresno, R.; Sajed, T.; Johnson, D.; Li, C.; Karu, N.; Sayeeda, Z.; Lo, E.; Assempour, N.; Berjanskii, M.; Singhal, S.; Arndt, D.; Liang, Y.; Badran, H.; Grant, J.; Serra-Cayuela, A.; Liu, Y.; Mandal, R.; Neveu, V.; Pon, A.; Knox, C.; Wilson, M.; Manach, C.; Scalbert, A. HMDB 4.0: The Human Metabolome Database for 2018. Nucleic Acids Res. 2018, 46 (D1), D608- 
D617. https://doi.org/10.1093/nar/gkx1089.

(20) Wishart, D. S.; Jewison, T.; Guo, A. C.; Wilson, M.; Knox, C.; Liu, Y.; Djoumbou, Y.; Mandal, R.; Aziat, F.; Dong, E.; Bouatra, S.; Sinelnikov, I.; Arndt, D.; Xia, J.; Liu, P.; Yallou, F.; Bjorndahl, T.; Perez-Pineiro, R.; Eisner, R.; Allen, F.; Neveu, V.; Greiner, R.; Scalbert, A. HMDB 3.0-The Human Metabolome Database in 2013. Nucleic Acids Res. 2012, 41 (D1), D801-D807. https://doi.org/10.1093/nar/gks1065.

(21) Wishart, D. S.; Knox, C.; Guo, A. C.; Eisner, R.; Young, N.; Gautam, B.; Hau, D. D.; Psychogios, N.; Dong, E.; Bouatra, S.; Mandal, R.; Sinelnikov, I.; Xia, J.; Jia, L.; Cruz, J. A.; Lim, E.; Sobsey, C. A.; Shrivastava, S.; Huang, P.; Liu, P.; Fang, L.; Peng, J.; Fradette, R.; Cheng, D.; Tzur, D.; Clements, M.; Lewis, A.; De Souza, A.; Zuniga, A.; Dawe, M.; Xiong, Y.; Clive, D.; Greiner, R.; Nazyrova, A.; Shaykhutdinov, R.; Li, L.; Vogel, H. J.; Forsythe, I. HMDB: A Knowledgebase for the Human Metabolome. Nucleic Acids Res. 2009, 37 (Database), D603-D610. https://doi.org/10.1093/nar/gkn810.

(22) Wishart, D. S.; Tzur, D.; Knox, C.; Eisner, R.; Guo, A. C.; Young, N.; Cheng, D.; Jewell, K.; Arndt, D.; Sawhney, S.; Fung, C.; Nikolai, L.; Lewis, M.; Coutouly, M.-A.; Forsythe, I.; Tang, P.; Shrivastava, S.; Jeroncic, K.; Stothard, P.; Amegbey, G.; Block, D.; Hau, D. D.; Wagner, J.; Miniaci, J.; Clements, M.; Gebremedhin, M.; Guo, N.; Zhang, Y.; Duggan, G. E.; MacInnis, G. D.; Weljie, A. M.; Dowlatabadi, R.; Bamforth, F.; Clive, D.; Greiner, R.; Li, L.; Marrie, T.; Sykes, B. D.; Vogel, H. J.; Querengesser, L. HMDB: The Human Metabolome Database. Nucleic Acids Res. 2007, 35 (Database), D521-D526. https://doi.org/10.1093/nar/gk1923.

(23) Ma, Y.; Kind, T.; Vaniya, A.; Gennity, I.; Fahrmann, J. F.; Fiehn, O. An in Silico MS/MS 
Library for Automatic Annotation of Novel FAHFA Lipids. J. Cheminform. 2015, 7 (1). https://doi.org/10.1186/s13321-015-0104-4.

(24) Aoyagi, R.; Ikeda, K.; Isobe, Y.; Arita, M. Comprehensive Analyses of Oxidized Phospholipids Using a Measured MS/MS Spectra Library. J. Lipid Res. 2017, 58 (11), 2229-2237. https://doi.org/10.1194/jlr.D077123.

(25) Fahy, E.; Subramaniam, S.; Murphy, R. C.; Nishijima, M.; Raetz, C. R. H.; Shimizu, T.; Spener, F.; van Meer, G.; Wakelam, M. J. O.; Dennis, E. A. Update of the LIPID MAPS Comprehensive Classification System for Lipids. J. Lipid Res. 2009, 50 (Supplement), S9S14. https://doi.org/10.1194/jlr.R800095-JLR200.

(26) Fahy, E.; Subramaniam, S.; Brown, H. A.; Glass, C. K.; Merrill, A. H.; Murphy, R. C.; Raetz, C. R. H.; Russell, D. W.; Seyama, Y.; Shaw, W.; Shimizu, T.; Spener, F.; van Meer, G.; VanNieuwenhze, M. S.; White, S. H.; Witztum, J. L.; Dennis, E. A. A Comprehensive Classification System for Lipids. Eur. J. Lipid Sci. Technol. 2005, 107 (5), 337-364. https://doi.org/10.1002/ejlt.200405001.

(27) Liebisch, G.; Vizcaíno, J. A.; Köfeler, H.; Trötzmüller, M.; Griffiths, W. J.; Schmitz, G.; Spener, F.; Wakelam, M. J. O. Shorthand Notation for Lipid Structures Derived from Mass Spectrometry. J. Lipid Res. 2013, 54 (6), 1523-1530. https://doi.org/10.1194/jlr.M033506.

(28) Sud, M.; Fahy, E.; Cotter, D.; Brown, A.; Dennis, E. A.; Glass, C. K.; Merrill, A. H.; Murphy, R. C.; Raetz, C. R. H. H.; Russell, D. W.; Subramaniam, S. LMSD: LIPID MAPS Structure Database. Nucleic Acids Res. 2007, 35 (Database), D527-D532. https://doi.org/10.1093/nar/gk1838.

(29) Voet, D.; Voet, J. G.; Pratt, C. W. Fundamentals of Biochemistry, 1st ed.; John Wiley \& 
Sons, Inc.: New York, NY, USA, 1998. 